\title{
EDITOR'S CHOICE
}

\section{Who are tomorrow's generalists?}

\author{
Jane Smith deputy editor, BMJ
}

People have been lamenting the decline of the general physician for decades-so it's good to see that the tide may now be turning.

In their Analysis article Robert M Wachter and Derek Bell describe the renaissance of hospital generalists on both sides of the Atlantic (doi:10.1136/bmj.e652). The reasons-growing numbers of older patients with complex multiple illnesses and curtailed hours for trainee doctors - are similar in the UK and the United States, but generalists have taken different forms in each country. In America hospitalists are responsible for the general medical care of patients during their entire stay in hospital, working alongside specialists. In the UK, physicians in acute medicine manage patients in the first 48-72 hours after an emergency admission, then hand over to specialists. The evidence suggests that both models reduce lengths of stay and improve outcomes for patients.

As I read this article I was wondering where geriatricians fitted in - aren't they meant to be generalist physicians skilled in managing older people with multiple illnesses? Editorialists $\mathrm{R}$ $\mathrm{M}$ Temple and colleagues confirm that geriatricians, as the largest group of medical consultants in the UK, do indeed perform that role, alongside physicians who combine general internal medicine with a specialty; but there aren't enough geriatricians, and few dually accredited trainees want to continue to practise general medicine when they become consultants (doi:10.1136/bmj.e2240). The editorialists see useful lessons from the hospitalist model, which "may help re-enthuse trainees and consultants."

Generalist medical skills seem to dominate NICE's latest guidance on improving care for people using NHS services (doi:10.1136/bmj.d6422). The essential requirements of care include recommendations on being prepared to raise sensitive issues (such as sexual activity, continence care, and end of life concerns), ensuring adequate nutrition and hydration, and ensuring pain relief, while the section on shared decision making recommends the best ways of discussing risks and benefits with patients. As Simon Eaton and colleagues say in their accompanying editorial, much of the guidance states the obvious and "it is a sad indictment of modern healthcare" that we need it (doi:10.1136/bmj.e2006). They advocate more sophisticated ways of measuring and reporting on patients' experiences and support for patients in managing their own chronic conditions.

Paul Truepenny, in this week's Patient Journey, illustrates what shared decision making and self management means: "I now expect my consultations to be within an equal partnership. My aim is to help healthcare staff to understand ... how this rare disease affects my life so that I can receive the best treatment and care available" (doi:10.1136/bmj.e1181). NICE couldn't have put it better.

Meanwhile, the letters section performs its invaluable role in telling readers how things really are. Eaton and colleagues may want more sophisticated measures of patient experience, but Lauren Brennan and colleagues suggest that there are some difficulties in getting the basics right (doi:10.1136/bmj.e2432). As part of an attempt to improve coding of episodes of care they reviewed the Hospital Episode Statistics and found that an increasing number of adults were attending child and adolescent psychiatry services, while over 3000 0-19 year olds attended geriatric outpatients in 2009-10. They were also surprised at the over 17000 men admitted to inpatient obstetric services.

"Although we applaud innovation, we suspect ... data errors [some] due to similarities in the main specialty codes."

Cite this as: BMJ 2012;344:e2495

๑ BMJ Publishing Group Ltd 2012 